\title{
Performance Evaluation of DWT-OFDM and DFT-OFDM in Multipath PLC Channel
}

\author{
Abdoreza Kiani \\ Department of Electrical Engineering, \\ Abadan Branch, \\ Islamic Azad University, \\ Abadan, Iran
}

\begin{abstract}
Power-line communication (PLC) carries data on a conductor that is also used simultaneously for AC electric power transmission or electric power distribution to consumers. Power-line communications systems operate by adding a modulated carrier signal to the wiring system. Different types of power-line communications use different frequency bands. Since the power distribution system was originally intended for transmission of AC power at typical frequencies of 50 or $60 \mathrm{~Hz}$, power wire circuits have only a limited ability to carry higher frequencies. Data rates and distance limits vary widely over many power-line communication standards. Lowfrequency (about 100-200 kHz) carriers impressed on highvoltage transmission lines may carry one or two analog voice circuits, or telemetry and control circuits with an equivalent data rate of a few hundred bits per second; however, these circuits may be many miles long. Higher data rates generally imply shorter ranges; a local area network operating at millions of bits per second may only cover one floor of an office building, but eliminates the need for installation of dedicated network cabling. Reliable and high speed communication over power lines requires a robust modulation scheme, like a Discrete Wavelet Transform (DWT) based modulation technique, hence the quality of data communication over the noisy in-home power line network can be improved. This paper investigates the performance of conventional Orthogonal Frequency Division Multiplexing (OFDM) and Discrete Wavelet Transform-based OFDM (DWT-OFDM) systems in the presence of background and impulsive noise in multipath power lines as a communication channels. The time and frequency localization properties of the wavelet transform mitigates narrowband and heavy impulsive noise in the power lines which results in performance improvement. Simulation results in terms of PAPR, PSD, and Bit Error Rate (BER) show that DWTOFDM is more robust against interference and multipath effects compared to DFT-OFDM, and increasing the length of the basis function improves BER and PAPR.
\end{abstract}

\section{Keywords}

DWT-OFDM,BPL,Multipath,Noise,BER,PAPR,PSD.

\section{INTRODUCTION}

Power line is a technology which uses existing electric power lines for data transmission. Electrical voltage is high voltage and alternatively slow, while a data signals voltage is small and alternatively fast. Since these waves have different frequencies, there is no cross interference when combing them. The overlapping signals are divided and the data signal is extracted by the PLC adaptor. This makes it possible to send data signals through power lines.
A universal in-home networking system can be easily and conveniently built as PLC technology transforms wall poweroutlets into information outlets.
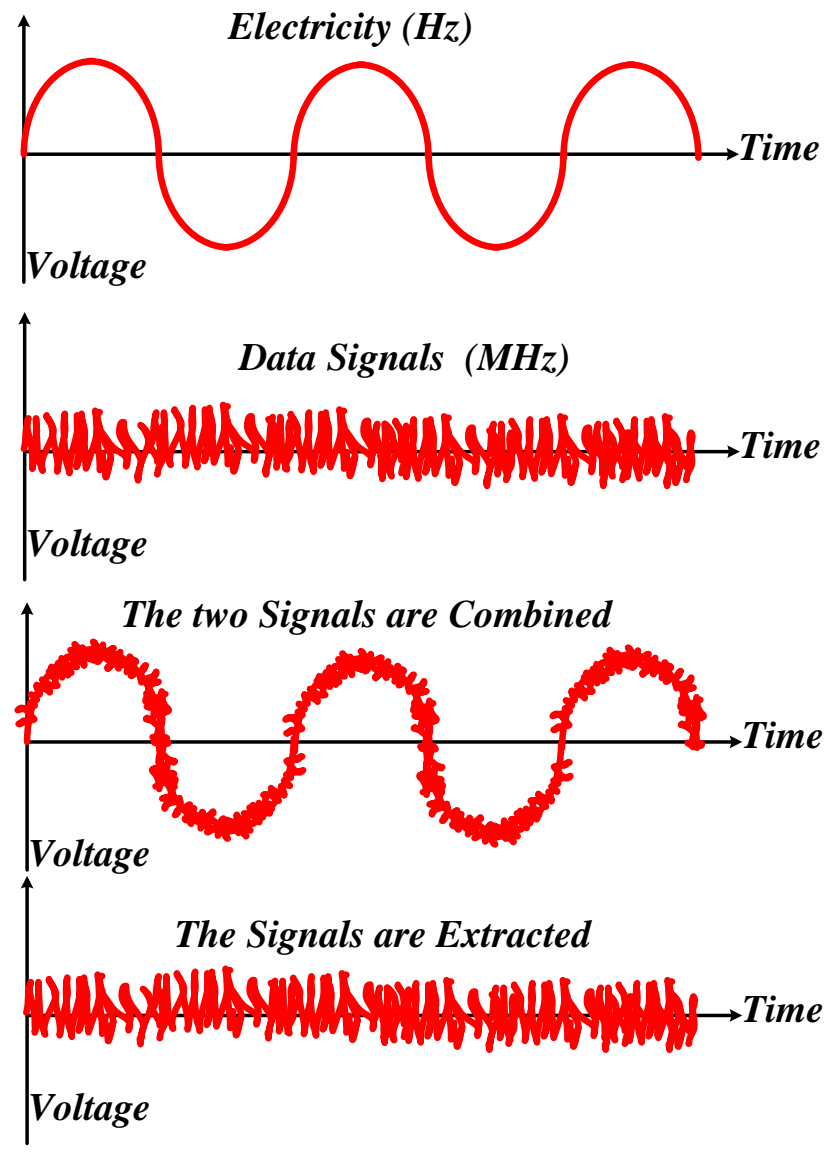

Figure 1. The combination of electrical and data signals.

In recent years, Broadband over Power Lines (BPL) technology, with applications such as high-speed internet access, Internet Protocol Television (IPTV), Voice over Internet Protocol (VoIP) and creating wired Local Area Networks (LAN) within the residential and commercial buildings, has gained more attention. BPL works on a similar principle to DSL technology. Computer network information can be transmitted over the lines using signaling frequencies higher than the electrical (or voice in the case of Digital Subscriber Line (DSL)) signals. Taking advantage of otherwise unused transmission capability of the wires, computer data can be sent back and forth across the BPL 
network with no disruption to power output in the home. The main advantage of implementing power line communications is that it needs no extra infrastructure, which can be expensive and time-consuming. However, power lines as communication channels, have their own complexities so that they are different from conventional communication channels such as telephone lines and wireless channels. The main drawback of PLC is that the power lines network was not originally designed for supporting data transmission. Also these lines are very noisy and propagation of signals over them exhibit strong Inter Symbol Interference (ISI) and frequency selective fading due to multipath propagation [1].

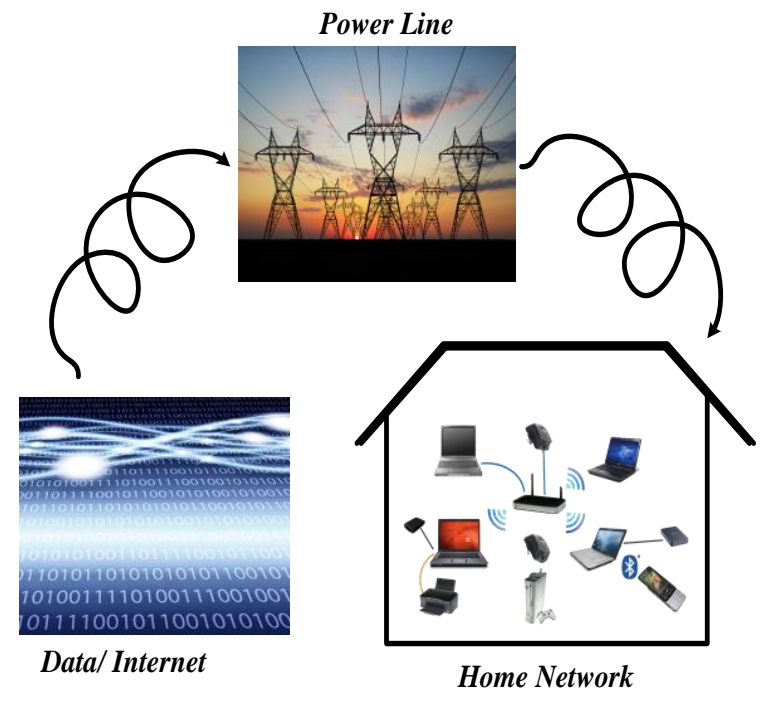

Figure 2. Broadband over Power Lines (BPL)

Multicarrier Modulations (MCM) are receiving increasing attention for their capability to cope with channels characterized by severe ISI without the use of complex equalizers. Conventional Orthogonal Frequency Division Multiplexing (OFDM) based on Discrete Fourier Transform (DFT) is the most common MCM method in which the orthogonality between subcarriers can prevent Inter-Carrier Interference (ICI) [2].

Discrete Wavelet Transform-based OFDM (DWT- OFDM) offers an alternative to the well-established DFT-OFDM as an efficient MCM technique. The incentive to use DWT rather than DFT in OFDM is to provide better spectral roll-off and to remove the need for the $\mathrm{CP}$ [2-3-4]. In the conventional OFDM, the pulse shaping filter is a rectangular window. As the rectangular window has large spectral side-lobes, there is a large spectral leakage. DWT -OFDM has a better performance regarding this leakage.

To study channel characteristics, an accurate channel model is an essential requirement. The basic model introduced in the time domain, due to the multipath nature of the power line, is Philipps's channel model [5-6]. Afterward, Zimmerman and Dostert proposed a time domain model which not only was according to the multipath effect, but also considering extra attenuation factor which increased the accuracy of Philipps's model [7]. In addition, Gali and Banwell introduced a model based on Multi-conductor Transmission Line (MTL) theory in the frequency domain [8-9]. The main drawback of this model is that the whole information of transmission line, such as the types of cables and their properties, topology and impedance at the end of the line, have to be known and any defect in the initial values, will reduce accuracy. In this paper, the performance of a PLC channel in different conditions is evaluated. Here, Zimmermann's channel model is utilized. Also a simple and effective noise model is used based on IEEE P1901 standard [10]. In [2-3] DFT-OFDM and Wavelet-OFDM with Cosine Modulated Filter Bank (CMFB) structure are compared. Following studying and simulating selected model for channel and noise in Section II, the Bit Error Rate (BER) and Peak to Average Power Ratio (PAPR) performances of DFT-OFDM and DWT-OFDM employing wavelet packet structure are carried out in multipath channel under impulsive noise and background noise in Section III. Finally, section IV concludes the paper.

\section{POWER LINE MODELING}

\section{A. Channel model}

Transfer function of transmission lines depends on many factors. Type of wires used in the distribution network with different characteristic impedances and lengths of wiring, topology, and types of loads which are connected to the network are the main factors. In these structures, high frequency signals are not distributed in a single path and will be damaged from reflection of the delayed and attenuated signals due to impedance mismatch in electrical networks. These reflections, which are called echo, give rise to frequency selective channel property. So PLC is modeled as a multipath frequency selective channel. Measurements show that the channel properties do not change very fast because of long channel coherence time compared with the transmitted symbol duration. Therefore, the channel can be considered as a quasi stationary channel [5]. With regard to these properties, after studying proposed types of channel models, Zimmermann's channel model based on superposition of $N$ paths, has been utilized, since it is a straightforward and accurate method. The frequency transfer function with an additional coefficient is given by

$$
H(f)=\sum_{i=1}^{N} \underbrace{g_{i}}_{\begin{array}{c}
\text { weighting } \\
\text { factor }
\end{array}} \underbrace{e^{-\left(a_{0}+a_{1} f^{k}\right) d_{i}}}_{\begin{array}{c}
\text { attenuation } \\
\text { portion }
\end{array}} \underbrace{e^{-j 2 \pi f \frac{d_{i}}{V_{p}}}}_{\begin{array}{c}
\text { delay } \\
\text { portion }
\end{array}}
$$

where $d_{i} / V_{p}$ is time delay in each path ( $d_{i}$ is the length and $v_{p}$ is the speed of light to pass through the insulation). $a_{1} a_{0}$ and $k$ are attenuation offset, attenuation coefficient, and attenuation power, respectively. Parameter values which are used for simulation is based on [7] and are shown in Table I.

Table 1parameters Of The 15-Path Model

\begin{tabular}{|c|c|c|c|c|c|}
\hline \multicolumn{7}{|c|}{ Attenuation Parameters } \\
\hline \multicolumn{2}{|c|}{$\boldsymbol{k}$} & \multicolumn{2}{|c|}{$\boldsymbol{a}_{\boldsymbol{0}}$} & \multicolumn{2}{c|}{$\boldsymbol{a}_{\boldsymbol{I}}$} \\
\hline \multicolumn{7}{|c|}{ Path Parameters } \\
\hline $\boldsymbol{7}$ & $\boldsymbol{g}_{\boldsymbol{i}}$ & $\boldsymbol{d}_{\boldsymbol{i}}[\boldsymbol{m}]$ & $\boldsymbol{i}$ & $\boldsymbol{g}_{\boldsymbol{i}}$ & $\boldsymbol{d}_{\boldsymbol{i}}[\boldsymbol{m}]$ \\
\hline & & & & & \\
\hline 1 & 0.029 & 90 & 9 & 0.071 & 411 \\
\hline 2 & 0.043 & 102 & 10 & -0.035 & 490 \\
\hline 3 & 0.103 & 113 & 11 & 0.065 & 567 \\
\hline & & & & & \\
4 & -0.058 & 143 & 12 & -0.055 & 740 \\
\hline
\end{tabular}




\begin{tabular}{|c|c|c|c|c|c|}
\hline \multicolumn{7}{|c|}{ Attenuation Parameters } \\
\hline \multicolumn{2}{|c|}{$\boldsymbol{k}$} & \multicolumn{2}{|c|}{$\boldsymbol{a}_{\boldsymbol{0}}$} & \multicolumn{2}{|c|}{$\boldsymbol{a}_{\boldsymbol{I}}$} \\
\hline 5 & -0.045 & 148 & 13 & 0.042 & 960 \\
\hline 6 & -0.040 & 200 & 14 & -0.059 & 1130 \\
\hline 7 & 0.038 & 260 & 15 & 0.049 & 1250 \\
\hline 8 & -0.038 & 322 & & & \\
\hline
\end{tabular}

\section{B. Modeling types of noise}

Besides signal distortion, due to cable losses and multipath propagation, noise is the most crucial factor influencing high speed digital communication over power lines. Unlike many other communication channels, the PLC does not represent an Additive White Gaussian Noise (AWGN) environment. In the frequency range from some hundred $\mathrm{KHz}$ up to $20 \mathrm{MHz}$ it is mostly dominated by narrow-band interference and impulsive noise [11]. The additive noise in a power-line system is divided in five categories which are grouped into two classes namely the generalized background and the impulsive noise [11-12]. The background noise includes colored background noise and noise due to the narrowband disturbers, which usually remain stationary over periods of minutes or sometimes even for hours. Periodic impulsive noise asynchronous or synchronous to the mains frequency and aperiodic impulsive noise asynchronous to the mains frequency, all together constitute the impulsive noise. The Power Spectral Density (PSD) of this type of noise can reach values of more than $50 \mathrm{~dB}$ above the background noise. The periodic asynchronous variations are similar to narrowband noise, whereas the other type can vary in durations of microseconds or milliseconds. The total background noise $N_{B}$ can be represented by

$N_{B}(f)=N_{C B}(f)+N_{N D}(f)$

where $N_{C B}$ and $N_{N D}$ represent the colored background noise and the noise caused by narrowband disturbers, respectively. The PSD of colored noise can be expressed by

$N_{C B}(f)=N_{0}+N_{1} e^{-\left(f / f_{1}\right)}$

where $N_{0}$ (in $\mathrm{dBm} / \mathrm{Hz}$ ) is the constant noise power density, $f_{l}$ (in $\mathrm{MHz}$ ) and $N_{l}$ are the parameters of the exponential function. For a residential environment, we used $N_{0}=N_{1}=$ 35 and $f_{1}=3.6$ in simulation [11]. The noise due to narrowband disturbers is given by

$N_{N D}(f)=\sum_{k=1}^{N} A_{k} e^{-\left(f-f_{0, k}\right)^{2} / 2 B_{k}^{2}}$

where $N$ is the total number of the disturbers, $f_{0}$ and $B_{k}$ (in $\mathrm{MHz}$ ) are the centre frequency and the bandwidth of the narrowband disturber, respectively, while $A_{k}$ in $\mathrm{dBm} / \mathrm{Hz}$ is its amplitude. The parameter $N$ follows a normal distribution and for the bandwidth $B_{k}$, the exponential distribution is applied. The last parameter, $A_{k}$, follows a uniform distribution for 0$10 \mathrm{MHz}$ and a normal distribution for $10-20 \mathrm{MHz}$. The statistical properties regarding all component parameters are taken into account and used in computer simulation. By this way, the real conditions on a PLC channel can be portrayed in the most precise way.

Fig. 3 shows the PSD of the colored background and narrowband noise up to $20 \mathrm{MHz}$. The asynchronous impulsive noise is a serious source of impairment for intra-building PLC since it is much stronger and therefore causes severe deterioration to the system's performance.

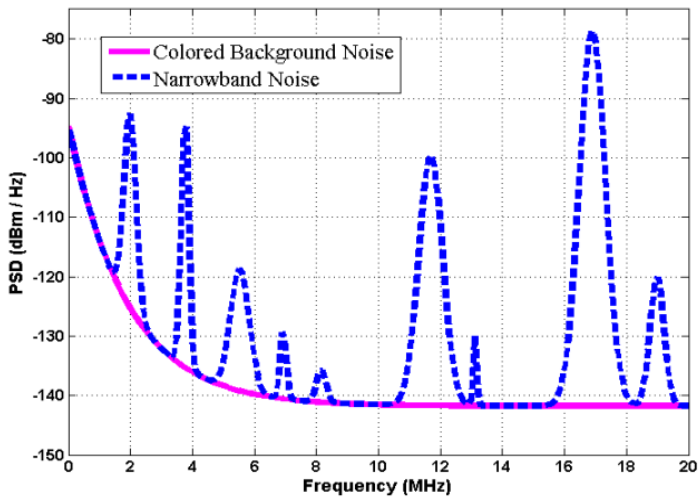

Figure 3. PSD of Colored Background and Narrowband Noise

Thus, when it comes to calculations, only this type is considered as the system's impulsive noise. The time domain analysis of impulse noise, shown in Fig. 4, is based on the amplitude $A$, impulse width, $t_{\mathrm{w}}$, and inter-arrival times, $t_{I A T}$ [13]. The last parameter is considered to be the most crucial one since it determines how frequent the impulses occur at the channel. The behavior of the impulse is expressed by impulse train

$N_{\text {imptrain }}(t)=\sum_{i=1}^{N} A_{i} \cdot \operatorname{imp}\left(\frac{t-t_{\text {arr }, i}}{t_{w, i}}\right)$

where $\mathrm{A}, t_{\mathrm{w}}$, and $t_{I A T}$ are random variables whose statistical properties are obtained by measurements [12-13-14].

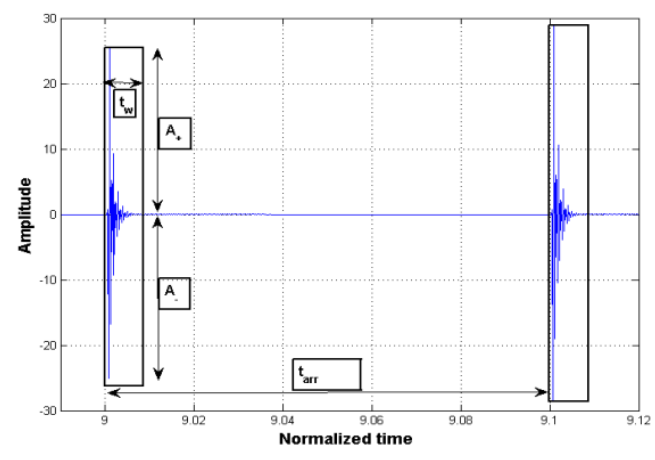

Figure 4. Impulsive Noise in time domain

\section{BLOCK DIAGRAMS OF SYSTEM AND SIMULATION RESULTS}

Transceiver systems are shown in Fig. 5. In DFT-OFDM system, the data bit stream is first mapped onto QAM constellation to form a complex symbol followed by S/P. Then it is modulated onto orthogonal subcarriers using IDFT. After P/S, a Cyclic Prefix (CP) (that is $25 \%$ of each symbol in practical systems) is wrapped to the symbols. Then the signals are passed through the power-line as a communication channel, followed by three noises. At the receiver, the CP is discarded. The resulting signal is demodulated to recover the original data bits.

DWT is a class of generalized Fourier transforms with basis function being localized well both in the time and frequency 
domains. They are constructed by means of Quadrature Mirror Filter (QMF) pairs [14-15]. It has been shown that DWTOFDM is more robust to narrowband interference and multipath propagation loss, than DFT-OFDM [16]. In DWTOFDM transmitter, the incoming signal is first converted from serial to parallel. In the case of DWT-OFDM, the number of iterations can be expressed by

Number of iterations $=\log _{2}$ ( Number of subcarriers)

Fig. 6 shows DWT and inverse DWT (IDWT) blocks. IDWT (as the synthesis filter bank) and DWT (as the analysis filter bank) are used in place of IDFT and DFT, respectively, at the transmitter and receiver. Any iteration of IDWT up-samples two signals and filters, one with a High Pass (HP) Finite Impulse Response (FIR) filter and the other one with a Low Pass (LP) FIR filter [17].

Consequently, DWT-OFDM does not require $\mathrm{P} / \mathrm{S}$ in the transmitter and S/P in the receiver. In our study, wavelets such as Daubechies (db) and Symlet (sym) are evaluated. When analysis bank is exchanged with the synthesis bank, the system will be still a Perfect Reconstruction (PR) [18]. Fundamentally, DFT-OFDM and DWT-OFDM have many similarities as both use orthogonal waveforms as subcarrier. The main difference between DFT-OFDM and DWT-OFDM is on the shape of the subcarrier and in the way they are created. One important property of wavelet is that the waveforms being used in general are longer than the transform duration of each symbol [19-20]. This causes DWT-OFDM symbols to overlap in the time domain. Longer waveforms allow better frequency localization of DWT-OFDM's subcarriers while in DFT-OFDM the rectangular shape of DFT window generates large side-lobes. Fig. 7-a and Fig. 5-b illustrate the spectra of 8 adjacent subcarriers for DWT(db16)OFDM and DFT-OFDM transceiver, respectively.
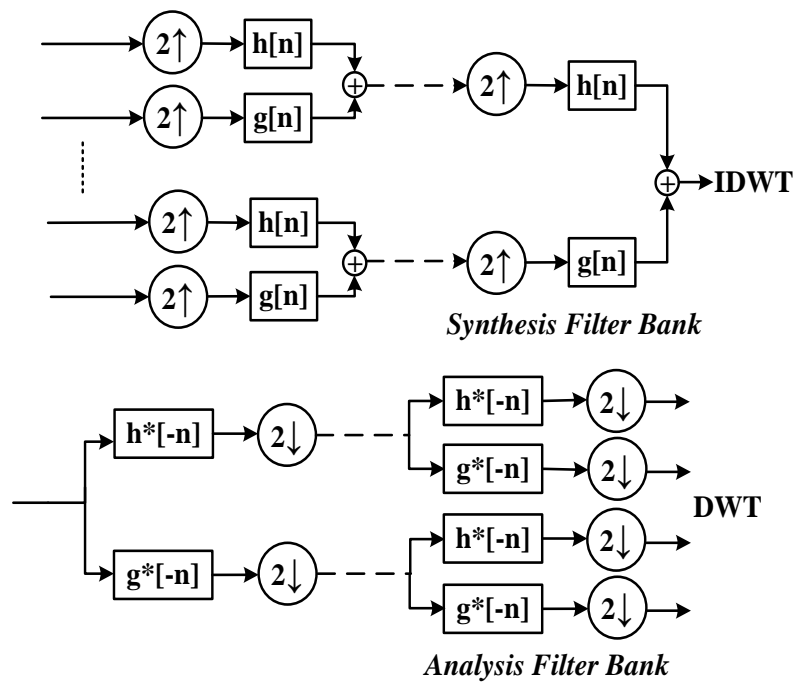

Figure 5. DWT and IDWT Block Diagrams

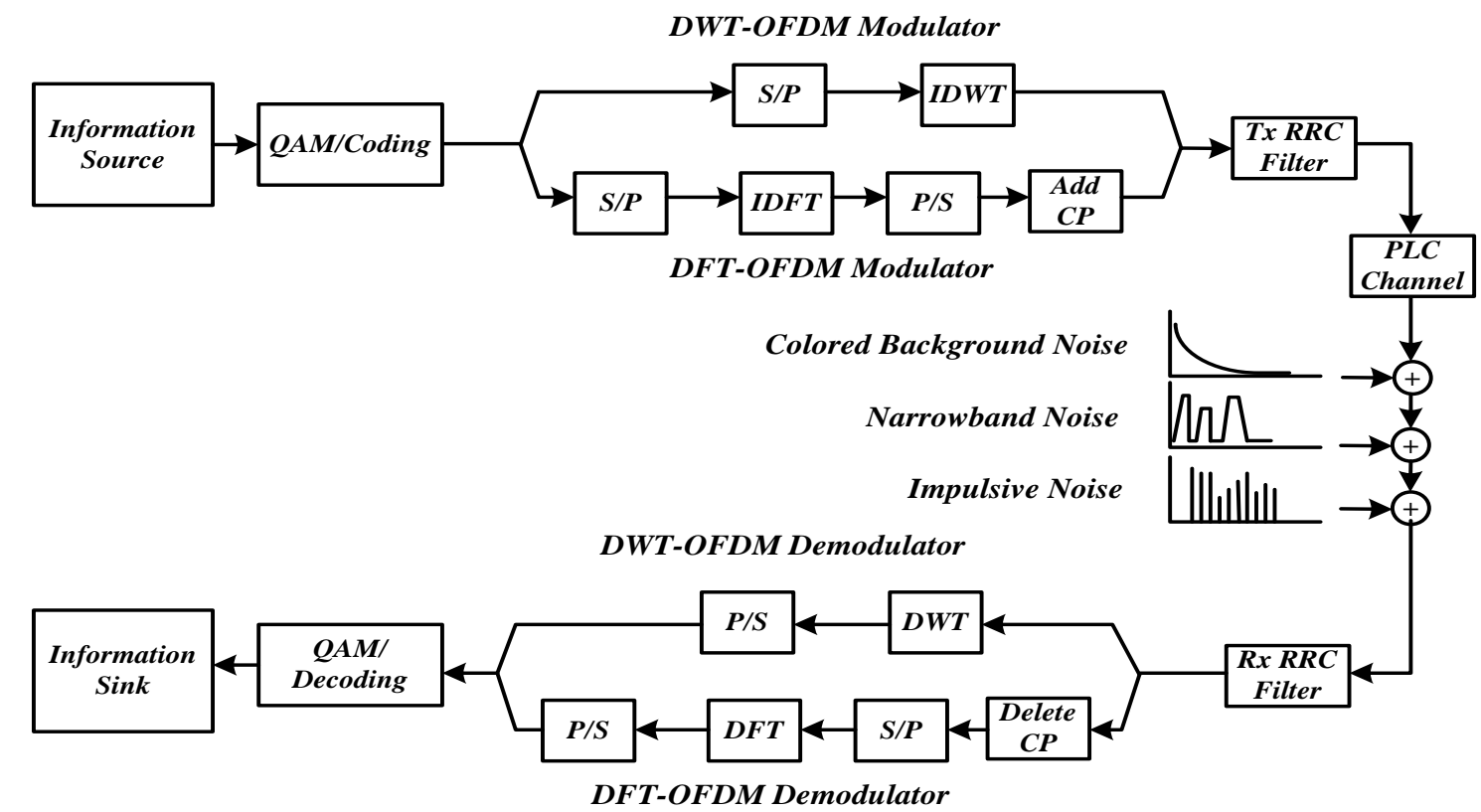

Figure 6. DFT and DWT Transceiver

Adding guard intervals and $\mathrm{CP}$, severely decrease spectral efficiency of DFT-OFDM. Now we show the performance of both systems in presence of background and impulse noise model on a 15-path power-line channel. The 4-QAM modulation scheme is used along with Convolutional Coding (CC) of code rate $1 / 2$. 


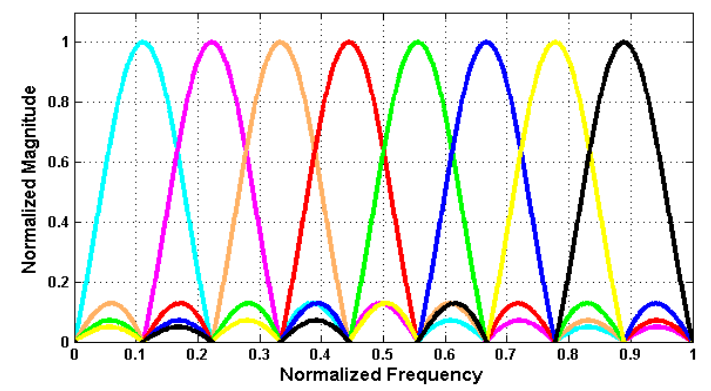

Figure 7-a. Spectrum of OFDM subcarrier.

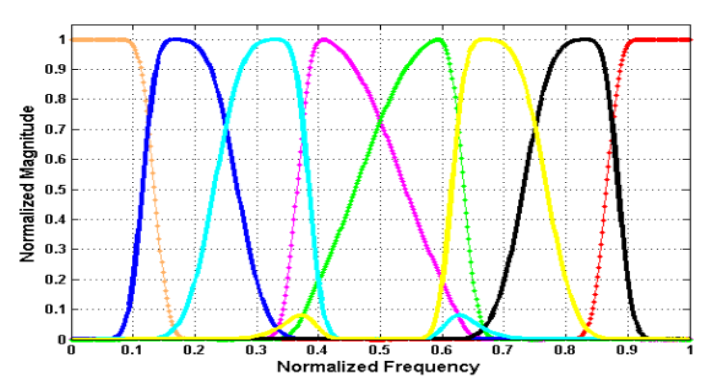

Figure 7-b. Spectrum of DWT(db16)-OFDM subcarrier.

Fig. 8 shows the simulation results in terms of BER versus the energy per bit to noise power spectral density ratio $\left(E_{b} /\right.$ $N_{o}$ ). The sym16 basis function shows $2 \mathrm{~dB}$ improvement in BER plot compared to DFT-OFDM and has better performance compared to Daubechies families. Since increasing filter length can reduce side-lobes and interference between subcarriers, so the BER performance improves with increasing the filter length.

One of the main drawbacks of OFDM is its high PAPR. PAPR is defined as the ratio between the maximum power in OFDM symbol to the average power of the same OFDM symbol as indicated by

$$
P A P R=\frac{\max |x(t)|^{2}}{E\left[|x(t)|^{2}\right]}
$$

where $E[$.$] denotes expectation. PAPR depends linearly on$ the number of subcarriers, but in systems with a large number of subcarriers, the probability of a symbol with a large PAPR is small and vice versa. This leads to use Cumulative Distribution Function (CDF) to describe PAPR distribution. The result can be interpreted as an ICI in the system [21]. In general, PAPR is evaluated from the discrete time samples by oversampling. PAPR can take values in a range that is proportional to the number of subcarriers. In this study, the DFT-OFDM and DWT-OFDM schemes with 64 subcarriers, each modulated with QPSK, were compared in terms of CDF. Fig. 9 shows that DWT(db16)-OFDM has a better PAPR performance in comparison to DFT-OFDM and other filters. As a rectangular window in DFT-OFDM has large spectral side-lobes, there is a large spectral leakage.

This could pose a problem in some applications. For example, in some wired systems that transmission of the PSD of downstream signal needs to fall below a threshold in the frequency bands of upstream transmission to avoid interference, the PSD should be attenuated in amateur radio bands to allow egress emission control. Fig. 10-a and Fig .10b show that Symlet family can reduce the spectral leakage more than db8, coif5 and DFT-OFDM.

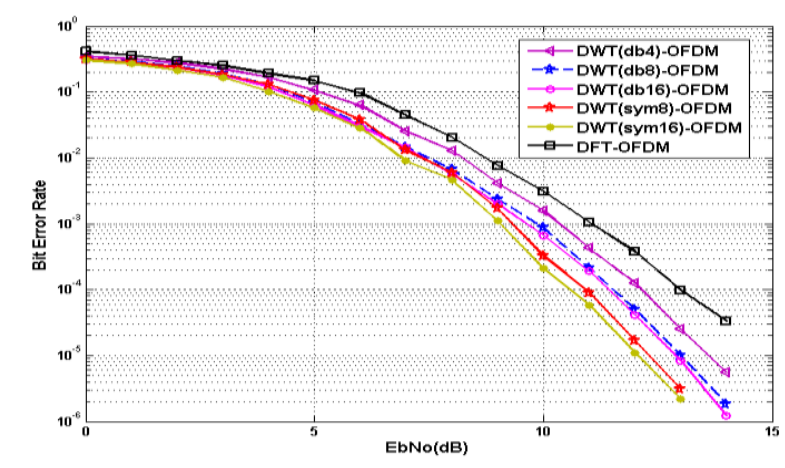

Figure 8. Performance of DFT-OFDM and DWT-OFDM.

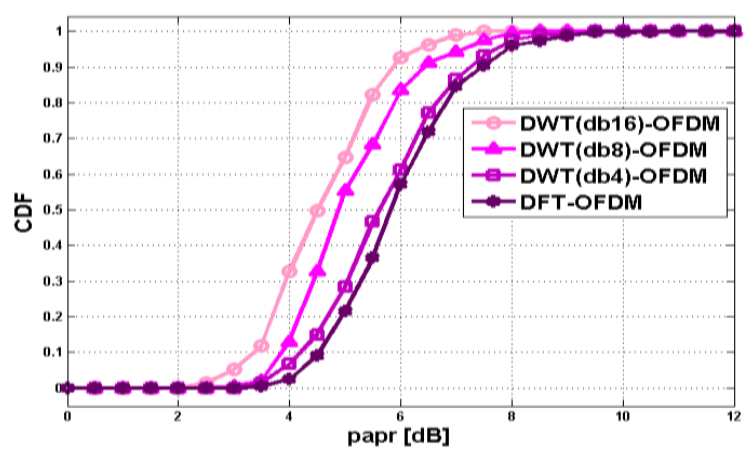

Figure 9. CDFs of the PAPR for different schemes.

\section{CONCLUSION}

In this paper the BER, PAPR, and PSD performances of DFTOFDM and DWT-OFDM in the presence of background and impulsive noise on multipath power line channel were evaluated. According to simulation results, it was found that the BER and PSD performances of DWT-OFDM are better than those of DFT-OFDM in the 15-path power line channel. In Daubechies and Symlet families, when the length of the filter was increased, the BER performances were increased. The results showed that sym16 for the DWT-OFDM system achieved a better BER compared to DFT-OFDM. Also, some DWT-OFDM schemes showed superior PAPR performances than the DFT-OFDM schemes.

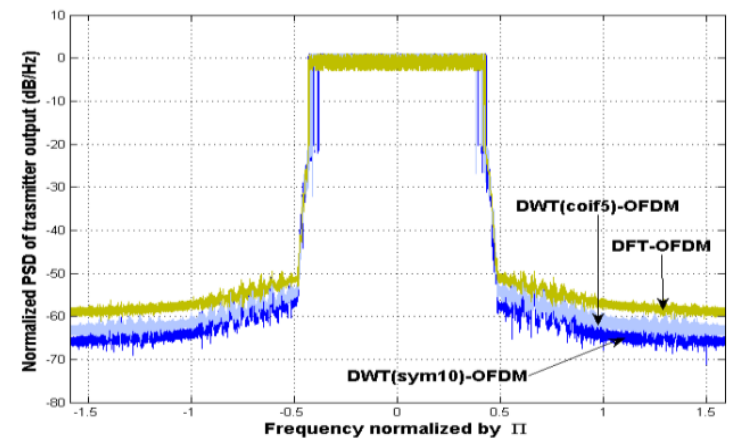

Figure 10-a. PSD comparison of DWT(coif5)-OFDM, DWT(sym10)-OFDM and DFT-OFDM. 


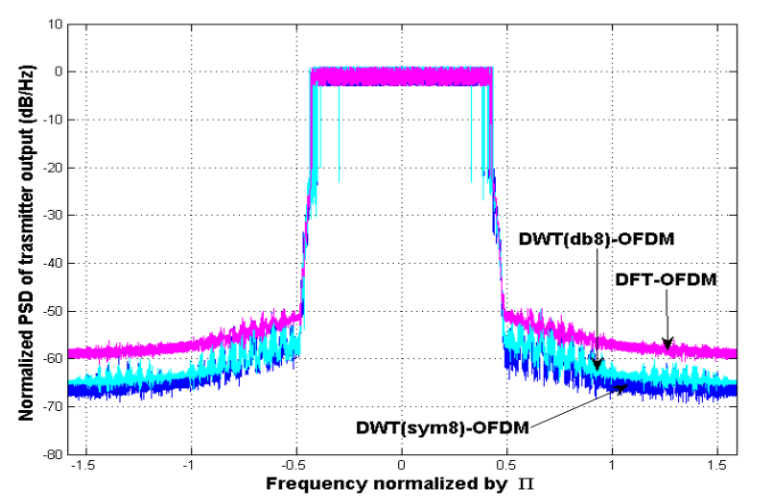

Figure 10-b. PSD comparison of DWT(db8)-OFDM, DWT(sym8)-OFDM and DFT-OFDM.

\section{REFERENCES}

[1] S. Baig and M. J. Mughal, "Multirate signal processing techniques for high-speed communication over power lines," IEEE Commun. Mag., vol. 47, no. 1, pp.70-76, Jan 2009.

[2] S. Galli, H. Koga, and N. Kodama, "Advanced signal processing for PLCs: Wavelet-OFDM," in Proc. IEEE Int. Symp. on Power Line Commun. and Its Applicat. 2008 (ISPC'08), pp. 187-193, Apr 2008.

[3] A. Farhang, M. Molavi, and B. Farhang Boroujeny, "Wavelet-OFDM versus Filtered-OFDM in Power Line Communication Systems," 5th Int. Symp. on Telecommun., pp. 691-694, Dec 2010.

[4] Z. Deng,W. Guan, J. Huang, D. Zou, and Yuetao Ge, "Wavelet-based Multi-Carrier Modulation on Power Line Communication," IEEE Int. conf. on wireless commun., networking and mobile computing, sept. 2009.

[5] H. Philipps, "Modeling of powerline communication channels," in Proc. 3rd Int. Symp. Power-Line Communications and its Applications (ISPLC'99), pp. 14-21, Mar. 1999.

[6] H. Philipps, "Development of a Statistical Model for Powerline Communication Channels", in Int. Symp. on Power Line Commun. (ISPLC), Ireland, pp. 153-160, Apr 2000.

[7] M. Zimmermann and K. Dostert, "A multi-path signal propagation model for the power line channel in the high frequency range," in Proc. 3rd Int. Symp. Power-Line Communications and its Applications (ISPLC'99), pp. 45-51, Mar. 1999.

[8] T. Banwell and S. Gali, "A Novel Approach to the Modeling of the Indoor Powerline Channel -Part I: Circuit Analysis and Companion Model," IEEE Trans. Power Del.,vol. 20, no.2, pp. 655-663, Apr 2005.

[9] T. Banwell and S. Galli, "A Novel Approach to the Modeling of the Indoor Powerline Channel -Part II:
Transfer Function and Its Properties," IEEE Trans. Power Del., vol. 20, no. 3, pp. 1869-1878, July 2005.

[10]IEEE. , "Standard for broadband over power line networks: medium access control and physical layer specifications," IEEE P1901, Sep. 2010.

[11] N. Andreadou and N. Pavlidou, "Modeling the noise on the OFDM powerline communications system," IEEE Trans. Power Del., vol. 25, no. 1, pp. 150-157, Jan. 2010.

[12] D. Benyoucef, "A New Statistical Model of the Noise Power Density Spectrum for Powerline Communication," 7th Int. Symp. on Power Line Commun. and Its Applicat, pp. 136-141, 26-28, Mar. 2003.

[13]M. Zimmermann and K. Dostert, "Analysis and Modeling of Impulsive Noise in Broad-Band Power line Communications," IEEE Trans. Electromagn. Compat., vol. 44, no. 1, pp. 249-258, Feb 2002.

[14] R. Lindsey, "Wavelet packet modulation: a generalized method for orthogonally multiplexed communications," in IEEE 27th Southeastern Symp. on System Theory, pp. 392-396, Mar 1995.

[15]D. Daly, C. Heneghan, A. Fagan, and M. Vetterli, "Optimal wavelet packet modulation under finite complexity constraint," in Proc. ICASSP, vol. 3, pp. 2789-2792, May 2002.

[16] P. Kaewmanee, R. F.Ormondroyd, C. R. Walters, "Resilient adaptive wavelet packet modulation scheme for Used in Time and Frequency Selective Channels Using the Best Tree Search Algorithm,"2004 IEEE Military Commun. Conf., pp. 1566-1571, Milom, Oct. 31 2004-Nov. 32004.

[17] P.P.Vaidyanathan, "A theory for multiresolution signal decomposition: The wavelet representation," IEEE Trans .Pattern Anal. Machine Intell, vol.11, pp.674-693, Jul 1989.

[18] G.Strang, T.Nquyen, Wavelets and Filter Banks, Wellesley-Cambridge Press, 1996.

[19] M.Vetterli, J. Kovacevic, Wavelets and Subband Coding.Prentice HallPTR, Englewood Cliffs, New Jersey, 1995.

[20]I.Daubechies, Ten Lectures on Wavelets, Philadelphia:SIAM, 1992

[21] A. Kiani, G. Baghersalimi and B. Zanj. "Performance Assessment of DFT-OFDM and DWT-OFDM Systems in the Presence of the HPA Nonlinearity," in Proc. IEEE Int. conf. on Telecommun. (ConTEL), pp. 273-278, Jun 2011. 